\title{
Erratum to: Flare-Shaped Acoustic Anomalies in the Water Column Along the Ecuadorian Margin: Relationship with Active Tectonics and Gas Hydrates
}

\author{
Francois Michaud, ${ }^{1,2}$ Jean-Nö̈l Proust, ${ }^{3}$ Alexandre Dano, ${ }^{2}$ Jean-Yves Collot, ${ }^{2,4}$ \\ Grâce Daniella Guiyeligou, ${ }^{2}$ María José Hernández Salazar, ${ }^{5}$ Gueorgui Ratzov, ${ }^{2}$ Carlos Martillo, ${ }^{2,3,6,7}$ \\ Hugo Pouderoux,${ }^{3}$ Laure Schenini, ${ }^{2}$ Jean-Frederic Lebrun, ${ }^{8}$ and Glenda Loayza ${ }^{6,7}$
}

\section{Erratum to: Pure Appl Geophys \\ DOI 10.1007/s00024-015-1230-7}

Due to technical problems for all authors of this paper the given names and the family names had been switched. The correct names are:

Francois Michaud, Jean-Noël Proust, Alexandre Dano, Jean-Yves Collot, Grâce Daniella Guiyeligou, María José Hernández Salazar, Gueorgui Ratzov, Carlos Martillo, Hugo Pouderoux, Laure Schenini, Jean-Frederic Lebrun, and Glenda Loayza

1 Univ. Pierre et Marie Curie, UPMC, CNRS, IRD, Observatoire de la Côte d'Azur, Géoazur UMR 7329, 250 rue Albert Einstein, 06560 Sophia Antipolis, Valbonne, France. E-mail: micho@geoazur.unice.fr

2 Univ. Nice Sophia Antipolis, CNRS, IRD, Observatoire de la Côte d'Azur, Géoazur UMR 7329, 250 rue Albert Einstein, 06560 Sophia Antipolis, Valbonne, France.

3 Géosciences Rennes, CNRS, Université de Rennes 1, Campus de Beaulieu, 35042 Rennes Cedex, France.

4 Investigador Prometeo, Instituto Geofísico, Escuela Politécnica Nacional, Ladrón de Guevara E11-253, Aptdo.2759 Quito, Ecuador.

5 Departamento de Geología, Escuela Politécnica Nacional, Ladrón de Guevara E11-53, Quito, Ecuador.

6 Facultad de Ingenieria en Ciencias de la Tierra, Escuela Superior Politécnica del Litoral, km 30.5 Vía Perimetral Campus "Gustavo Galindo", Guayaquil, Ecuador.

7 Instituto Oceanográfica de la Armada de Ecuador (INOCAR), Avenida 25 de Julio vía Puerto Marítimo, Base Naval Sur, Guayaquil, Ecuador.

8 Univ. des Antilles et de la Guyane, Campus de Fouillole, 97159 Pointe Á Pitre Cedex, Guadeloupe, France. 